\title{
Applications of Operation research in the Field of IOT
}

\author{
Navleen Kaur ${ }^{1}$,Kavita $^{2}$ \\ 1,2 Department of Mathematics,Chandigarh University, Gharuan \\ ${ }^{1}$ Navleen.appsci@ cumail.in, ${ }^{2}$ kavita.cse@ cumail.in
}

Article History: Received: 11 January 2021; Accepted: 27 February 2021; Published online: 5 April 2021

ABSTRACT: These days Internet of Things (IoT) increased an extraordinary consideration structure specialist, since it turns into a significant innovation that guarantees a shrewd individual life, by permitting an interchanges between objects, machines and each thing together with people groups. IoT speaks to a framework which comprises a things in reality, and sensors joined to or consolidated to thesethings, associated with the Internet through wired and remote system structure.In this paper I have represented the various application of the operation research in the field of IoT.

\section{Keywords: Operations Research,Internet of Things}

\section{Introduction}

Operations research $(\mathrm{OR})$ is an analytical procedureto solve problem and decision-making which is used by the management of organizations. In operations research, problems are divided intodifferent components and then by using mathematical analysis, solution of each component is found out .In similar way it works in the field of IoT.Without using the quantitative and qualitative techniques of Operation Research,IoT cannot be completed.Hence OR(operation research ) plays a vital role in the field of IoT(internet of things)

\section{What is iot}

People today have made significant progress in front of the early-man ages when they needed to physically take part in the consummation of every one of their needs and necessities. Today life has gotten less complex, quicker just as more helpful than it at any point was. Have you at any point figured what it resembles to have all the gadgets, individuals, protests just as living and non-living elements to be associated over a typical system?

The Internet of Things known as IoT, is a method for associating all the gadgets and articles around us into a common cloud network. Through the appearance of this innovation, we will have the option to impart just as send/get information over this system without the requirement for any human mediation. You will be stunned to realize that while this mechanical change is by all accounts to some degree unimaginable at the present time; the execution of IoT towards making a savvy world has just been set into movement. The development of Internet of Thingstechnology is expanding so quickly that a significant number of the IT technology speculators have begun putting resources into IoT stocks market.

The Internet of Things

\section{From connecting devices to human value}

01

Device connection lat itevicmes of cemrinctivity imbeririest intelluenro

Data sensing Capiore dida Burvars and Legh Blor actu

\section{Communication} Tous on acoess Nebwotis, cloukt, otge Deta tranesort

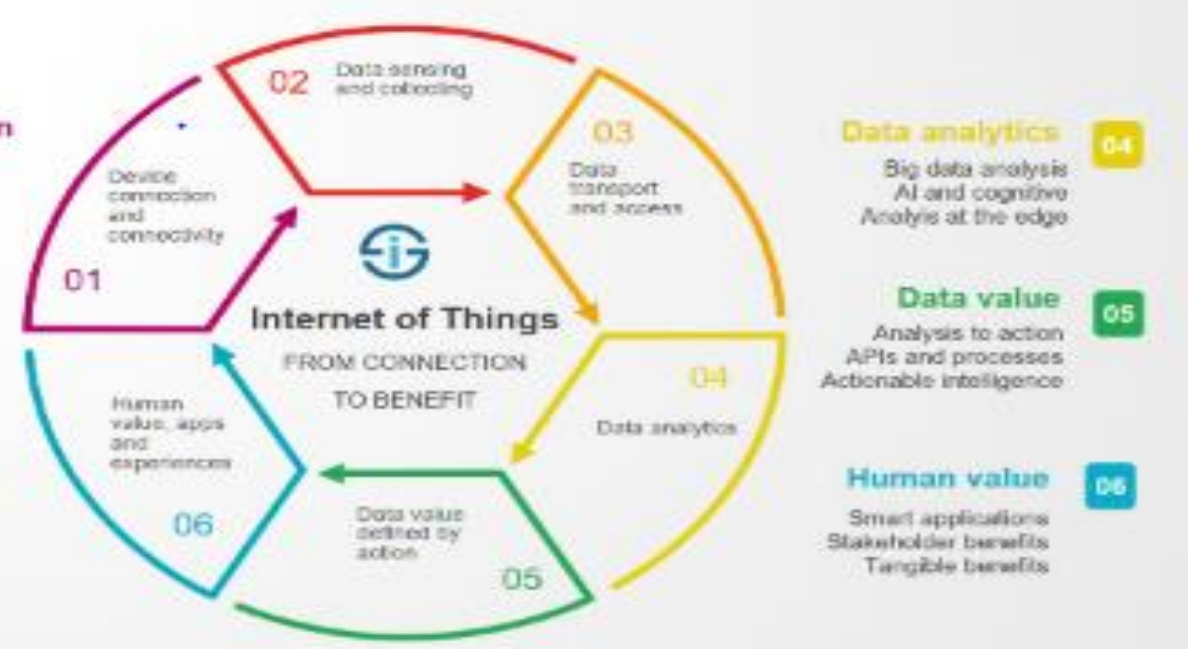

Fig-1-Internet of Things 


\section{Research challenge in iot}

IoT enable us to communicate with each other through the devices like smart phone, computer etc. We use the internet to share multimedia and information.As food, Internet is also a necessary part of common man's life. Now in these days increasing use of IoT also brings challenge for us to do more research in this field. These are the challenges of design, multi-technology infrastructure, management, networking etc. In this paper we have discussed some tools of operation research to meet the challenges

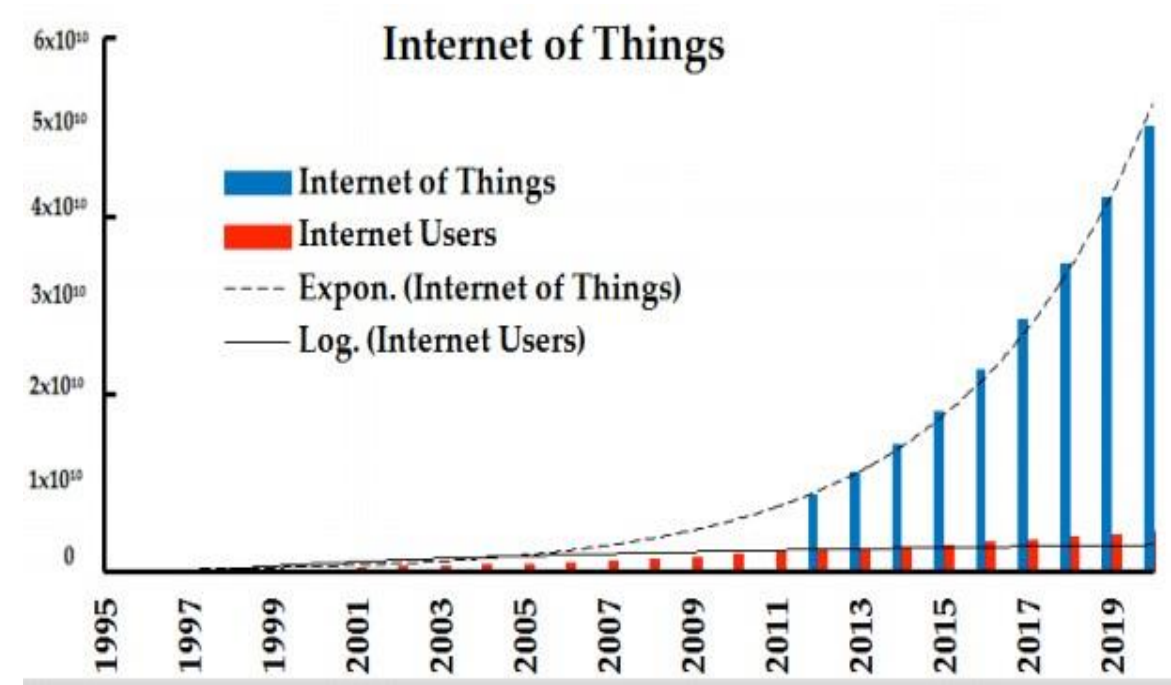

.(Data from http://www.internetlivestats.com/internet-users/)

\section{APPLICATION OF OR (OPERATION RESEARCH) IN iot(INTERNET OF THINGS)}

In this section ,I am discussing the various tools and technologies of operation research which are necessary to meet the required challenges of ToT

\subsection{Game Theory :}

Game theory is used to choose the best strategy out of the available strategies to maximize the winning.this approach is used in multi tasking like data distribution to optimize it. Now we are using LTE, $5 \mathrm{G}$, the optimization of these data distribution is investigated through game theory.

\subsection{Math Programming :}

In operation research we find optimal solution of linear, nonlinear, integer programming, dynamic programming. This programming is used as networking in IoT.By using this technology we can optimize (minimize/maximize)the use of networking.

\subsection{Simulation :}

A real world process can be copied from computer using Simulation. For this ,simulation requires a mathematical model of the real world which must contains the behavior of the system. Many simulation model are available for networking which are used in IoT. For example, a pilot learned simulation training to react to a life threatening loss of engine. Simulation here related to the environmental effects on IoT.

\subsection{Stochastic (Marcov) process :}

Many mathematical processes are used to test the reliability of test .Marcov process is one of them. In IoT this theory is used to test the performance of system and used to improve this. It also helps to evaluate the performance of components during operation.It is also applied to test the service quality and the management of data.

\subsection{Graph Theory :}


Graph theory is a pictorial representation of vertices and edges in which vertices are connected with each other through the edge(edges).Which is similar as networking. In IoTwe use three types of networks known as topological networks, data-functional networks, and domio-functional networks.Graph theory is used to combine this networks .it is also used in cell phone networking, computer networking, sensor networking.

\subsection{Queuing Theory :}

Queuing theory is a branch of operation research which represents different mathematical model. These models are used to estimate service time, average waiting time etc.InIoT emails, messages are sent, and received through a proper network so the queuing theory is a perfect tool to measure the required results. In electronic mail the model of queuing theory which is used is known as IMAP( Inter Mail Access Protocol)

\subsection{CPM(Critical Path Method) :}

Critical path is used to visualize the project in the graphical form. It defines the tasks which are most important.it also reduce the time of the project and identify the critical activities in which more attention is required. In the similar way it is used in the networking of IoT. It helps the signal to follow those paths in which less time is consumed. Networking of IoT is tottaly followed the management as CPM.

\subsection{Agent based Modelling :}

In IoT lots of messages ,emails, signals move at the same time, which creates traffic. To Reduce the traffic load agent based modeling is used which works as smart objent interaction.

\section{Conclusion}

TheIot has become an interesting cocept with sensory hardware, smart phones, communication protocols along with social and cyber networks. It also include lots of complexities whose range is from mechanisim, information management, communication, presentation and interaction with in the IoT.No doubt there are lots of existing model which are capable to design and solve problems related to IoT.but as the use of it increasing day by day lot of research work is required., This paper presents a synthetic reaction-inspired computational model utilizing the ideas of graphs and game, which endeavors to address the complexities related with the perception, demonstrating, communication, investigation and deliberation of data in the IoT.

\section{References}

1. Borgia, E.,2016. Special Issue on Ïnternet of Things: Research Challenges and Solutions (editorial). 89, p1-4.

2. Brandenburger, A.M.; Nalebuff, B.J., 1995. "The right game: Use game theory to shape strategy". Harvard Bus., 73, p57-71.

3. Gubbi, J.; Buyya, R.; Marusic, S.; Palaniswami, M., 2013. "Internet of Things (IoT): A vision, architectural elements, and future directions". Future Gener.Comput. Syst. 29, p1645-1660.

4. Lessman, K.; Riecken, M.; O’Connor, M.J., 2016. "Modeling \& Simulation of the Internet of Things and Cyber Physical Systems for Cybersecurity". In Proceedings of the Simulation InnovationWorkshop, Orlando, FL, USA.

5. Manoj Dubey,2019 “'Internet of Things: Mathematical Relevance', International Conference on Recent Advances in Interdisciplinary Trends in Engineering \& Application

6. Ribeiro, R.A.; Falcao, A.; Mora, A.; Fonseca, J.M.,2014. "A fuzzy information fusion algorithm based on multi-criteria decision making”. Knowl.-Based Syst., 58, p23-32.

7. Rose, K.; Eldridge, S.; Chapin, L., 2015. “The internet of things: An overview”. In Understanding the Issues and

8. Challenges of a More Connected World; The Internet Society (ISOC):Washington, DC, USA, pp. $1-50$. 